Research Article

\title{
Enhancement of Capacitated Transportation Problem in Fuzzy Environment
}

\author{
Hamiden Abd El-Wahed Khalifa, ${ }^{1}$ Pavan Kumar ${ }^{(D)},{ }^{2}$ and Majed G. Alharbi ${ }^{3}$ \\ ${ }^{1}$ Operations Research Department, Faculty of Graduate Studies for Statistical Research, Cairo University, and Giza, Egypt \\ ${ }^{2}$ Mathematics Division, School of Advanced Science \& Languages, VIT Bhopal University, Sehore, MP 466114, India \\ ${ }^{3}$ Department of Mathematics, College of Science and Arts, Methnab, Qassim University, Saudi Arabia
}

Correspondence should be addressed to Pavan Kumar; pavankmaths@gmail.com

Received 30 July 2020; Accepted 19 September 2020; Published 1 October 2020

Academic Editor: Ping-Feng Pai

Copyright ( $\odot 2020$ Hamiden Abd El-Wahed Khalifa et al. This is an open access article distributed under the Creative Commons Attribution License, which permits unrestricted use, distribution, and reproduction in any medium, provided the original work is properly cited.

\begin{abstract}
This research work aims to study a capacitated transportation problem (CTP) with penalty cost, supplies, and demands represented by hexagonal fuzzy numbers. Based on ranking function, the supplies and demands are converted to the crisp form. Through the use of the $\alpha$-level, the problem is converted into interval linear programming. To optimize the interval objective function, we define the order relations represented by policy maker's choice between intervals. The maximization (minimization) problem considering the interval objective function is transformed to multiobjective optimization problem based on order relations introduced by the preference of policy makers between interval profits (costs). A numerical example is given for illustration and to check the validity of the suggested approach.
\end{abstract}

\section{Introduction}

Transportation problem (TP) is considered as a particular case of linear programming (LP) problem, where the decision maker wants to minimize the cost of the distributing product from $m$ sources or origins to $n$ distributions, and their capacities are $u_{1}, u_{2}, \ldots, u_{m}$ and $v_{1}, v_{2}, \ldots, v_{n}$, respectively. Additionally, there is a provision for charging the penalty $c_{i j}$, which consists of transportation of a unit of product from origin $i$ to destination $j$. This penalty is generally of the form of cost, delivery time of safety, delivery, etc. A variable $x_{i j}$ denotes the unknown quantity to be shipped from origin $i$ to destination $j$ [1].

There are so many difficulties in dealing a TP. One of them is that the parameters in the problem formulation are not constants but fluctuating and uncertain. Transportation or shipping problem (TP) involves determining the amount of goods or items to be transported from a number of origins to a number of destinations. TP is a specific case of linear programming (LP) problems, and a special algorithm has been developed to solve it. Oheigeartaigh [2] presented a fuzzy transportation algorithm with some applications. Vajda [3] developed an algorithm for a multiindex TP which is the extension of the distribution modification methodology. Pandian and Anuradha [4] discussed in their research work, a new method to find an optimal solution of STP related to the principle of zero-point methodology, which was first developed by Pandian and Natarajan [5].

Zadeh [6] introduced the fuzzy set theory that has been applied to solve numerous practical models, including financial engineering and risk management. The reason is that this theory permits the decision maker to investigate and deal the uncertain elements present. Then, the imperfect knowledge of the returns on the assets and the uncertainty involved in the behavior of financial markets may also be introduced by means of fuzzy quantities and/or fuzzy constraints. Fuzzy numerical data can be represented by means of fuzzy subsets of the real line, known as fuzzy numbers. Dubois and Prade [7] extended the use of algebraic operations on real numbers to fuzzy numbers by use of a fuzzification principle. Sakawa and Yano [8] introduced the concept of $\alpha$-Pareto optimality of fuzzy parametric 
programs. Chanas and Kuchta [9] studied a concept for determining the optimal solution of the transportation problem with fuzzy cost coefficients. Pandian and Natrajan [5] developed an optimal solution method for more or less type solution to a TP with mixed constraints, in fuzzy environment. A method for solving fuzzy TP was developed by Basirzadeh [10].

Kaur and Kumar [11] described a novel methodology to solve fuzzy TP by applying the ranking function. Kaur and Kumar [12] also developed a novel methodology to solve TP. They applied the generalized trapezoidal fuzzy numbers. Zangiabadi and Maleki [13] applied the fuzzy goal programming method for determining the multiple objective TP with some nonlinear membership functions. Khalaf [14] presented a solution method for fuzzy transportation problems. Dutta and Kumar [15] presented the fuzzy goal programming approach to solve the inventory model. They considered the fractional programming model in their study. Kumar and Dutta [16] studied a multiobjective fractional inventory model with price-dependent demand rate in fuzzy environment. Shu and Deng [17] presented the fuzzy mathematical programming approach to heterogeneous multiattribute decision-making problems. They considered the interval-valued intuitionistic fuzzy truth degrees' approach in their mathematical model. Shu and Dong [18] investigated the interval-valued intuitionistic fuzzy mathematical programming method to solve the hybrid multicriteria group decision-making problem. They considered the interval-valued intuitionistic fuzzy truth degrees. Hunwisai and Kumam [19] proposed a method for solving a fuzzy transportation problem through the robust ranking technique. Kumar et al. [20] proposed a novel computational procedure to solve the fuzzy Pythagorean TP, where they further developed the interval basic feasible solution, followed by the existing optimality method for obtaining the transportation cost.

Bit [21] applied the fuzzy programming approach with hyperbolic membership functions for linear multiobjective capacitated transportation problem (MOCTP). In their research, they considered the supply and demand constraints as equality type; capacity restrictions on each route are specified, and the objectives are noncommensurable and conflicting in nature so as to obtain efficient solutions as well as the best compromise solution. The capacitated transportation model is important to handle the capacity constraints efficiently, and it occurs frequently in applications. A TP with capacity restriction is an LP problem. A basic solution to a CTP may contain more than $m+n-2$ positive values on account of capacity constraints that are additional to the $m+n-2$ independent equations [22]. Dahiya and Verma [23] introduced a class of the CTP with bounds on total availabilities at sources and total destination requirements. Sharma et al. [24] studied capacitated two-stage time minimization TP in which the total availability of a homogeneous product at various resources is more than the total requirement of the same at destination. Shu and Jiu [25] presented the possibility linear programming under fuzziness. They considered the trapezoidal fuzzy numbers in their research work. Shu and Deng [26] studied Atanassov's intuitionistic fuzzy programming method for heterogeneous multiattribute group decision-making. They considered Atanassov's intuitionistic fuzzy truth degrees' approach.

Shu et al. [27] studied the intuitionistic fuzzy programming technique to solve the group decision-making. They considered the interval-valued fuzzy preference relations in their mathematical model. Shu et al. [28] discussed the hesitant fuzzy mathematical programming method. They considered an application in the area of hybrid multicriteria group decision-making with hesitant fuzzy truth degrees. Gupta et al. [29] formulated a new model of MOCTP considering the mixed constraints in which few objective functions are linear, while the others are assumed to be fractional. This situation is conflicting by nature. Ahmadi [30] applied the simplex method for bounded variables to obtain the optimal solution of the CTP. Shu and Dong [31] presented their research work on pythagorean fuzzy mathematical programming method, where they considered the multiattribute group decision-making problems. Dong and Wan [32] proposed a novel fuzzy linear programming method considering the acceptance degree of fuzzy constraints violated. They incorporated the trapezoidal fuzzy numbers to investigate the same. Dong and Shu [33] presented a new approach to solve the multiobjective linear programming problem under fuzziness.

The rest of the paper is organized as follows: In Section 2, we introduce the basic concepts and results related to fuzzy numbers, hexagonal fuzzy numbers, and its $\alpha$-level. Section 3 formulates capacitated transportation problem in fuzzy environment. Section 4 proposes solution procedure for obtaining $\alpha$-best compromise solution. In Section 5, a numerical example to illustrate the efficiency of the solution procedure is given. In Section 6, the results and discussions are presented. In the end, some concluding remarks are mentioned in Section 7.

\section{Preliminaries}

This section introduces some of basic concepts and results related to fuzzy numbers, hexagonal fuzzy numbers and their arithmetic operations, and its $\alpha$-cut.

Definition 1. A fuzzy set $\widetilde{P}$ defined on the set of real numbers $\mathbb{R}$ is said to be fuzzy numbers if its membership function, $\mu_{P}^{\sim}(x): \mathbb{R} \longrightarrow[0,1]$, has the following properties:

(1) $\mu_{\mathcal{P}}(x)$ is an upper semicontinuous membership function

(2) $\widetilde{P}$ is convex fuzzy set, i.e., $\mu_{\tilde{P}}(\delta x+(1-$

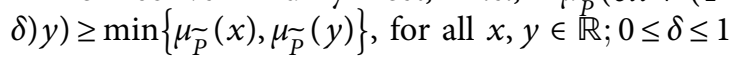

(3) $\widetilde{P}$ is normal, i.e., $\exists x_{0} \in \mathbb{R}$ for which $\mu_{P}\left(x_{0}\right)=1$

(4) $\operatorname{Supp}(\widetilde{P})=\left\{x \in \mathbb{R}: \mu_{P}(x)>0\right\}$ is the support of $\widetilde{P}$, and the closure $\mathrm{cl}(\operatorname{Supp}(\widetilde{P}))$ is a compact set

Definition 2 (see [34-36]). A fuzzy number $\widetilde{P}_{H}$ is a hexagonal fuzzy number denoted by $\widetilde{P}_{H}=\left(p_{1}, p_{2}, p_{3}\right.$, $\left.p_{4}, p_{5}, p_{6}\right)$, where $p_{1} \leq p_{2} \leq p_{3} \leq p_{4} \leq p_{5} \leq p_{6}$ are real 
numbers satisfying $p_{2}-p_{1} \leq p_{3}-p_{2}$, and $p_{5}-p_{4} \geq p_{6}-p_{5}$, if its membership function $\mu_{\tilde{P}_{H}}$ is given by

$$
\mu_{P_{H}}= \begin{cases}0, & x<p_{1}, \\ \frac{1}{2}\left(\frac{x-p_{1}}{p_{2}-p_{1}}\right), & p_{1} \leq x \leq p_{2}, \\ \frac{1}{2}+\frac{1}{2}\left(\frac{x-p_{2}}{p_{3}-p_{2}}\right), & p_{2} \leq x \leq p_{3}, \\ 1, & p_{3} \leq x \leq p_{4}, \\ 1-\frac{1}{2}\left(\frac{p_{5}-x}{p_{5}-p_{4}}\right), & p_{4} \leq x \leq p_{5}, \\ \frac{1}{2}\left(\frac{p_{6}-x}{p_{6}-p_{5}}\right), & p_{5} \leq x \leq p_{6}, \\ 0, & x>p_{6} .\end{cases}
$$

Definition 3 (see [34-36]). A hexagonal fuzzy number is defined as $\widetilde{P}_{H}=\left(A_{1}(u), B_{1}(v), B_{2}(v), A_{2}(u)\right)$, for $u \in[0,0.5]$ and $v \in[0.5,1]$, where

(i) $A_{1}(u)$ is a bounded left continuous nondecreasing function over $[0,0.5]$

(ii) $B_{1}(v)$ is a bounded left continuous nondecreasing function over $[0.5,1]$

(iii) $B_{2}(v)$ is a bounded left continuous nonincreasing function over $[1,0.5]$

(iv) $A_{2}(u)$ is a bounded left continuous nonincreasing function over $[0.5,0]$

The graphical representation of a hexagonal fuzzy number is shown is in Figure 1.

Definition 4 (see [34-36]). The $\alpha$-cut set of hexagonal fuzzy numbers $\widetilde{P}_{H}$ is referred as follows:

$$
\left(\widetilde{P}_{H}\right)_{\alpha}= \begin{cases}{\left[2 \alpha\left(p_{2}-p_{1}\right)+p_{1},-2 \alpha\left(p_{6}-p_{5}\right)+p_{6},\right.} & \alpha \in[0,0.5]] ; \\ {\left[2 \alpha\left(p_{3}-p_{2}\right)-p_{3}+2 p_{2},-2 \alpha\left(p_{5}-p_{4}\right)+2 p_{5}-p_{4}\right],} & \alpha \in[0.5,1] .\end{cases}
$$

Definition 5 (see [34-36]). Let $\widetilde{P}_{H}=\left(p_{1}, p_{2}, p_{3}, p_{4}, p_{5}, p_{6}\right)$ and $\widetilde{Q}_{H}=\left(q_{1}, q_{2}, q_{3}, q_{4}, q_{5}, q_{6}\right)$ be two hexagonal fuzzy numbers. The arithmetic operations on $\widetilde{P}_{H}$, and $\widetilde{Q}_{H}$ are

$$
\begin{aligned}
\widetilde{P}_{H} \oplus \widetilde{Q}_{H} & =\left(p_{1}+q_{1}, p_{2}+q_{2}, p_{3}+q_{3}, p_{4}+q_{4}, p_{5}+q_{5}, p_{6}+q_{6}\right), \\
\widetilde{P}_{H} \ominus \widetilde{Q}_{H} & =\left(p_{1}-q_{6}, p_{2}-q_{5}, p_{3}-q_{4}, p_{4}-q_{3}, p_{5}-q_{2}, p_{6}-q_{1}\right), \\
k \widetilde{P}_{H} & =\left(k p_{1}, k p_{2}, k p_{3}, k p_{4}, k p_{5}, k p_{6}\right), \quad k>0, \\
\widetilde{P}_{H} \odot \widetilde{Q}_{H} & =\left(\frac{p_{1}}{6} \delta_{q}, \frac{p_{2}}{6} \delta_{q}, \frac{p_{3}}{6} \delta_{q}, \frac{p_{4}}{6} \delta_{q}, \frac{p_{5}}{6} \delta_{q}, \frac{p_{6}}{6} \delta_{q}\right),
\end{aligned}
$$$$
\text { where } \delta_{q}=q_{1}+q_{2}+q_{3}+q_{4}+q_{5}+q_{6} \text {. }
$$

Definition 6. If $\widetilde{p}_{H}=\left(p_{1}, p_{2}, p_{3}, p_{4}, p_{5}, p_{6}\right)$ is a hexagonal fuzzy number, then its associated ordinary number is given by $\widehat{p}=\left(\left(p_{1}+p_{2}+p_{3}+p_{4}+p_{5}+p_{6}\right) / 6\right)$. The same ordinary number can be found by $\widehat{p}=\left(\left(p_{1}+p_{2}+2 p_{3}+\right.\right.$ $\left.\left.2 p_{4}+p_{5}+p_{6}\right) / 8\right)$.

Definition 7 (see [37]). An interval on $\mathbb{R}$ is defined as $A=$ $\left[a^{L}, a^{R}\right]=\left\{a:, a^{L} \leq a \leq a^{R}, a \in \mathbb{R}\right\}$, where $a^{L}$ and $a^{R}$ are left side limit and right limit of $A$, respectively.
Definition 8 (see [37]). The interval is also defined by $A=$ $a_{C}, a_{W}=\left\{a: a_{C}-a_{W} \leq a \leq a_{C}+a_{W}, a \in \mathbb{R}\right\}$, where $a_{C}=$ $(1 / 2)\left(a^{R}+a^{L}\right)$ is the center and $a_{W}=(1 / 2)\left(a^{R}-a^{L}\right)$ is the width of $A$.

\section{Problem Statement and Solution Concepts}

Consider the following CTP with fuzzy cost, supply, and demand as

$$
\left(\mathrm{P}_{1}\right) \min \widetilde{Z}_{H}=\sum_{i \in I} \sum_{j \in J}\left(\tilde{c}_{i j}\right)_{H} x_{i j}
$$

subject to

$$
\begin{aligned}
& \sum_{i \in I} x_{i j}(\leq,=, \geq)\left(\widetilde{a}_{i}\right)_{H}, \quad i \in I=1,2, \ldots, m, \\
& \sum_{i \in I} x_{i j}(\leq,=, \geq)\left(\widetilde{b}_{j}\right)_{H}, \quad j \in J=1,2, \ldots, n, \\
& l_{i j} \leq x_{i j} \leq u_{i j}, \\
& \text { integers, for all } i \in I, j \in J .
\end{aligned}
$$

Here, $x_{i j}$ is the quantity transported from source $i$ to destination $j,\left(\widetilde{c}_{i j}\right)_{H}$ is the cost of transporting one unit between source $i$ and destination $j,\left(\widetilde{a}_{i}\right)_{H} \pm 0(i \in I)$ is the supply of the source $i$, and $\left(\widetilde{b}_{j}\right)_{H} \pm 0(j \in J)$ is the demand of destination $j$. We assume that $\sum_{i \in I}\left(\widetilde{a}_{i}\right)_{H}=\sum_{j \in J}\left(\widetilde{b}_{j}\right)_{H}$. To make the problem consistent, we have $\sum_{i \in I} l_{i j}{ }^{\circ}\left(\widetilde{\mathrm{a}}_{\mathrm{i}}\right)$ ${ }_{H}, \sum_{i \in I} l_{i j}^{\circ}\left(\widetilde{b}_{\mathrm{j}}\right)_{\mathrm{H}}, \sum_{\mathrm{i} \in \mathrm{I}} \mathrm{u}_{\mathrm{ij}} \pm\left(\widetilde{\mathrm{a}}_{\mathrm{i}}\right)_{\mathrm{H}}$, and $\sum_{\mathrm{i} \in \mathrm{I}} \mathrm{u}_{\mathrm{ij}} \pm\left(\widetilde{\mathrm{b}}_{\mathrm{j}}\right)_{\mathrm{H}}$. 


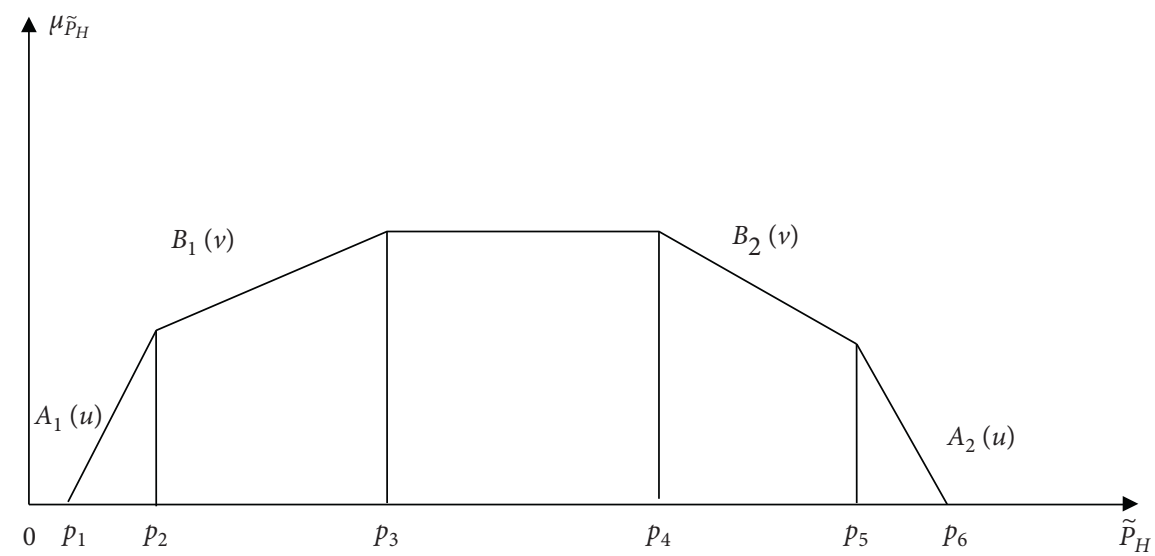

FIgURE 1: Graphical representation of a hexagonal fuzzy number.

Problem $\left(\mathrm{P}_{1}\right)$ is equivalent to

$$
\left(\mathrm{P}_{2}\right) \min \widetilde{Z}_{H}=\sum_{i \in I} \sum_{j \in J}\left(\widetilde{c}_{i j}\right)_{H} y_{i j}+\widetilde{U}_{H},
$$

subject to

$$
\begin{aligned}
& \sum_{i \in I} y_{i j}(\leq,=, \geq)\left(\widetilde{c}_{i}\right)_{H}, \quad i \in I=1,2, \ldots, m, \\
& \sum_{i \in I} y_{i j}(\leq,=, \geq)\left(\widetilde{d}_{j}\right)_{H}, \quad j \in J=1,2, \ldots, n, \\
& 0 \leq y_{i j} \leq u_{i j}-l_{i j}, \\
& \text { integers, for all } i \in I, j \in J,
\end{aligned}
$$

where $\tilde{U}_{H}=\sum_{i \in I} \sum_{j \in J}\left(\widetilde{c}_{i j}\right)_{H} l_{i j},\left(\widetilde{c}_{i}\right)_{H}=\left(\widetilde{a}_{i}\right)_{H}-\sum_{j \in J} l_{i j}$, and $\left(\tilde{d}_{j}\right)_{H}=\left(\tilde{b}_{j}\right)_{H}-\sum_{i \in I} l_{i j}$.

It is clear that there is a fuzzy feasible solution $x_{i j}=$ $y_{i j}+l_{i j}$ of problem $\left(\mathrm{P}_{1}\right)$ corresponding to the fuzzy feasible solution $y_{i j}$ of problem $\left(\mathrm{P}_{2}\right)$. Also, the fuzzy optimum value of problem $\left(\mathrm{P}_{1}\right)$ corresponding to the fuzzy feasible solution $x_{i j}=y_{i j}+l_{i j}$ is equal to the fuzzy optimum value of problem $\left(\mathrm{P}_{2}\right)$ corresponding to the fuzzy feasible solution $y_{i j}$. In addition, there is one-one and onto relation between fuzzy optimal solutions of $\mathrm{P}_{1}$ and $\mathrm{P}_{2}$. Thus, we consider the following problem instead of problem $\mathrm{P}_{1}$ as follows:

$$
\left(\mathrm{P}_{3}\right) \min \widetilde{Z}_{H}=\sum_{i \in I} \sum_{j \in J}\left(\widetilde{c}_{i j}\right)_{H} x_{i j}
$$

subject to

$$
\begin{aligned}
& \sum_{i \in I} x_{i j}(\leq,=, \geq)\left(\widetilde{a}_{i}\right)_{H}, \quad i \in I=1,2, \ldots, m, \\
& \sum_{i \in I} x_{i j}(\leq,=, \geq)\left(\widetilde{b}_{j}\right)_{H}, \quad j \in J=1,2, \ldots, n, \\
& 0 \leq x_{i j} \leq u_{i j}, \\
& \text { integers, for all } i \in I, j \in J .
\end{aligned}
$$

For a certain degree of $\alpha$ and based on the ranking of hexagonal fuzzy number in Definition 6, problem $\left(\mathrm{P}_{3}\right)$ can be rewritten as in the following nonfuzzy form

$$
\left(\mathrm{P}_{4}\right) \min Z=\sum_{i \in I} \sum_{j \in J} c_{i j} x_{i j}
$$

subject to

$$
x \in X=\left\{\begin{array}{cc}
\sum_{i \in I} x_{i j}(\leq,=, \geq) R\left(\left(\widetilde{a}_{i}\right)_{H}\right) & i \in I=1,2, \ldots, m \\
\sum_{I \in I} x_{i j}(\leq,=, \geq) R\left(\left(\widetilde{b}_{j}\right)_{H}\right) & j \in J=1,2, \ldots, n \\
c_{i j} \in\left(\left(\widetilde{c}_{i j}\right)_{H}\right)_{\alpha} & \alpha \in[0,1] \\
0 \leq x_{i j} \leq u_{i j} & \text { integers for all } i \in I, j \in J
\end{array}\right\} .
$$

Lemma 1. Problem $\left(P_{4}\right)$ is feasible if and only if $\left(\left(R\left(\left(\widetilde{a}_{i}\right)_{H}\right) R\left(\left(\widetilde{b}_{j}\right)_{H}\right)\right) / v\right) \leqq u_{i j}$, for all $i \in I, j \in J$, where $v=$ $\sum_{i \in I} R\left(\left(\tilde{a}_{i}\right)_{H}\right)=\sum_{j \in J} R\left(\left(\widetilde{b}_{j}\right)_{H}\right)$.
Proof. Similar to the proof introduced by [30].

From the properties of the $\alpha$-cut set, $\left(\widetilde{c}_{i j}\right)_{H}$ can be denoted by the interval confidence: 


$$
\left(\left(\widetilde{c}_{i j}\right)_{H}\right)_{\alpha}= \begin{cases}{\left[2 \alpha\left(c_{i j}^{2}-c_{i j}^{1}\right)+c_{i j}^{1},-2 \alpha\left(c_{i j}^{6}-c_{i j}^{5}\right)+c_{i j}^{6},\right.} & \alpha \in[0,0.5]] \\ {\left[2 \alpha\left(c_{i j}^{3}-c_{i j}^{2}\right)-c_{i j}^{3}+2 c_{i j}^{2},-2 \alpha\left(c_{i j}^{5}-c_{i j}^{4}\right)+2 c_{i j}^{5}-c_{i j}^{4}\right],} & \alpha \in[0.5,1] .\end{cases}
$$

Therefore, problem $\left(\mathrm{P}_{4}\right)$ may be written as

$$
\left(\mathrm{P}_{5}\right) \min Z=\sum_{i \in I} \sum_{j \in J}\left[\left(\left(\widetilde{c}_{i j}\right)_{H}\right)_{\alpha}^{L},\left(\left(\widetilde{c}_{i j}\right)_{H}\right)_{\alpha}^{R}\right] x_{i j}, \quad \alpha \in[0,1],
$$

subject to

Definition 9. $x \in X^{\prime}$ is an $\alpha$-solution of problem $\left(\mathrm{P}_{5}\right)$ if and only if there is no $\hat{x} \in X^{\prime}$ satisfies $Z(\hat{x}) \leq_{\mathrm{LR}} Z(x)$ or $Z(\widehat{x})<{ }_{\mathrm{CW}} Z$.

In order to specify Definition 9, let us define the order relation $\leq_{\mathrm{RC}}$ as follows:

$$
\begin{gathered}
A \leq{ }_{R C} B \Longleftrightarrow a_{R} \leq b_{R}, a_{C} \leq b_{C}, \\
A<{ }_{\mathrm{RC}} B \Longleftrightarrow A \leq{ }_{\mathrm{RC}} B, A \neq B .
\end{gathered}
$$

Proposition 1 (see [37]). It follows that

$$
\begin{aligned}
& A \leq_{\mathrm{RC}} B \Leftrightarrow A \leq_{\mathrm{LR}} B \text { or } A \leq_{\mathrm{CW}} B, \\
& A<{ }_{\mathrm{RC}} B \Leftrightarrow A \leq_{\mathrm{LR}} B \text { or } A<{ }_{\mathrm{CW}} B .
\end{aligned}
$$

According to Proposition 1, Definition 9 can be simplified as in Definition 10.

Definition 10. $x \in X^{\prime}$ is an $\alpha$-efficient solution of problem $\left(\mathrm{P}_{5}\right)$ if and only if there is no $\hat{x} \in X^{\prime}$ which satisfies $Z(\widehat{x}) \leq{ }_{\mathrm{RC}} Z(x)$.

The solution set of problem $\left(\mathrm{P}_{5}\right)$ can be obtained as the $\alpha$-efficient solution of the following multiobjective problem:

$$
\begin{aligned}
& \left(\mathrm{P}_{6}\right) \min \left(Z_{R}(x), Z_{C}(x)\right) \\
& \text { subject to } x \in X^{\prime} .
\end{aligned}
$$

Using the weighting Tchebycheff problem [38], problem $\left(\mathrm{P}_{6}\right)$ can be written in the following form:

$$
\left(\mathrm{P}_{6}\right) \min \psi \text {, }
$$

subject to $w_{1}\left[Z_{R}-\widehat{Z}_{R}\right] \leq \psi$,

$$
\begin{aligned}
& w_{2}\left[Z_{C}-\widehat{Z}_{C}\right] \leq \psi, \\
& x \in X^{\prime},
\end{aligned}
$$

where $w_{1}, w_{2} \geq 0 ; \widehat{Z}_{1}$ and $\widehat{Z}_{2}$ are the ideal targets.

Remark 1. Problem $\left(\mathrm{P}_{6}\right)$ may be rewritten as in the equivalent form: subject to

$$
\begin{aligned}
& w_{C}\left[Z_{C}-\widehat{Z}_{C}\right]-Z_{R}+\widehat{Z}_{R} \\
& x \in X^{\prime},
\end{aligned}
$$

where $w_{C} \geq 0 ; \widehat{Z}_{1}$ and $\widehat{Z}_{2}$ are said to be the ideal targets.

\section{Solution Procedure}

The steps of the solution procedure for solving the capacitated transportation problem in fuzzy environment can be summarized as follows:

Step 1: formulate problem $\left(\mathrm{P}_{3}\right)$

Step 2: estimate the ideal points $\widehat{Z}_{R}$ and $\widehat{Z}_{C}$ from the following relation:

$$
\widehat{Z}_{R}=\min Z_{R} \text {, }
$$

subject to $x \in X^{\prime}$ and

$$
\widehat{Z}_{C}=\min Z_{C}
$$

subject to $x \in X^{\prime}$

Step 3: calculate the individual maximum and minimum of each objective function subject to the given constraints for $\alpha=1$ and $\alpha=0$, respectively

Step 4: compute the weights from the relation

$$
\begin{gathered}
w_{R}=\frac{\overline{Z_{R}}-\underline{Z_{R}}}{\left(\overline{Z_{R}}-\underline{Z_{R}}\right)+\left(\overline{\bar{Z}_{C}}-\underline{Z_{C}}\right)}, \\
w_{C}=\frac{\overline{Z_{C}}-\underline{Z_{C}}}{\left(\overline{Z_{R}}-\underline{Z_{R}}\right)+\left(\overline{\bar{Z}_{C}}-\underline{Z_{C}}\right)},
\end{gathered}
$$

here $\overline{Z_{R}}, \overline{Z_{C}}$ and $Z_{R}, Z_{C}$ are the individual maximum and minimum of $Z_{R}$ and $Z_{C}$, respectively 
Step 5: ask the decision maker (DM) to specify $\alpha(0 \leq \alpha \leq 1)$,

Step 6: formulate the problem $\left(\mathrm{P}_{7}\right)$, and then applying the software GAMS to obtain the $\alpha$-optimal compromise solution, and hence the fuzzy cost.

Step 7: stop

\section{Numerical Example}

Consider the following fuzzy CTP:

$$
\min \widetilde{Z}_{H}=\sum_{i=1}^{3} \sum_{j=1}^{3}\left(\widetilde{c}_{i j}\right)_{H} x_{i j},
$$

subject to

$$
\begin{aligned}
& \sum_{j=1}^{3} x_{1 j}=\left(\widetilde{a}_{1}\right)_{H}, \\
& \sum_{j=1}^{3} x_{2 j}=\left(\widetilde{a}_{2}\right)_{H} \text {, } \\
& \sum_{j=1}^{3} x_{1 j}=\left(\tilde{a}_{3}\right)_{H}, \\
& \sum_{i=1}^{3} x_{i 1}=\left(\widetilde{b}_{1}\right)_{H^{\prime}} \\
& \sum_{i=1}^{3} x_{i 2}=\left(\widetilde{b}_{2}\right)_{H} \text {, } \\
& \sum_{i=1}^{3} x_{i 3}=\left(\tilde{b}_{3}\right)_{H^{\prime}} \\
& x_{i j} \in X^{\prime}=\left(\begin{array}{c}
x_{11}+x_{12}+x_{13}=13, x_{21}+x_{22}+x_{23}=14, x_{31}+x_{32}+x_{33}=16, \\
x_{11}+x_{21}+x_{31}=14, x_{12}+x_{22}+x_{32}=13, x_{13}+x_{23}+x_{33}=16 \\
2 \leq x_{11} \leq 5.5,5.5 \leq x_{12} \leq 9,4.5 \leq x_{13} \leq 12, \\
3 \leq x_{21} \leq 7,2 \leq x_{22} \leq 8,5 \leq x_{23} \leq 10, \\
1 \leq x_{31} \leq 5,4 \leq x_{32} \leq 9,2 \leq x_{33} \leq 7
\end{array}\right) \text {, }
\end{aligned}
$$

where

Supplies:

$$
\begin{aligned}
& \left(\widetilde{a}_{1}\right)_{H}=(8,9,11,14,16,20), \\
& \left(\widetilde{a}_{2}\right)_{H}=(9,10,12,15,18,20), \\
& \left(\widetilde{a}_{3}\right)_{H}=(8,11,14,18,20,25) .
\end{aligned}
$$

Demands:

$$
\left(\widetilde{c}_{i j}\right)_{H}=\left(\begin{array}{c}
(3,7,11,15,19,24,)(3,5,7,9,10,12)(11,14,17,21,25,30) \\
(3,5,7,9,10,12)(5,7,10,13,17,21)(7,9,11,14,18,22) \\
(7,9,11,14,18,22)(2,3,4,6,7,9)(5,7,8,11,14,17)
\end{array}\right)
$$


It is clear that

$$
\begin{aligned}
& R\left(\left(\tilde{a}_{1}\right)_{H}\right)=13, \\
& R\left(\left(\tilde{a}_{2}\right)_{H}\right)=14, \\
& R\left(\left(\tilde{a}_{3}\right)_{H}\right)=16, \\
& R\left(\left(\tilde{b}_{1}\right)_{H}\right)=14, \\
& R\left(\left(\tilde{b}_{2}\right)_{H}\right)=13, \\
& R\left(\left(\tilde{b}_{3}\right)_{H}\right)=16 .
\end{aligned}
$$

Suppose the decision maker selects $\alpha=0.85$, then

$$
\left(\left(\widetilde{c}_{i j}\right)_{H}\right)_{\alpha}=\left(\begin{array}{ccc}
{[9.8,16.2]} & {[6.4,9.3]} & {[16.1,22.2]} \\
{[6.4,14.2]} & {[10.4,15.2]} & {[10.4,15.2]} \\
{[10.4,15.2]} & {[3.7,6.3]} & {[11.1,11.9]}
\end{array}\right) .
$$

Step 3: solve the following problems individually:

$$
\begin{aligned}
& \underline{Z}_{R}=\min \left(\begin{array}{c}
16.2 x_{11}+9.3 x_{12}+22.2 x_{13}+14.2 x_{21}+15.2 x_{22} \\
15.2 x_{23}+15.2 x_{31}+6.3 x_{32}+11.9 x_{33}
\end{array}\right), \\
& \underline{Z}_{C}=\min \left(\begin{array}{c}
13 x_{11}+7.85 x_{12}+19.15 x_{13}+10.3 x_{21}+12.8 x_{22} \\
12.8 x_{23}+12.8 x_{31}+5 x_{32}+11.5 x_{33}
\end{array}\right),
\end{aligned}
$$

subject to

$$
\begin{aligned}
x_{i j} \in X^{\prime} & =\left(\begin{array}{c}
x_{11}+x_{12}+x_{13}=13, x_{21}+x_{22}+x_{23}=14, x_{31}+x_{32}+x_{33}=16, \\
x_{11}+x_{21}+x_{31}=14, x_{12}+x_{22}+x_{32}=13, x_{13}+x_{23}+x_{33}=16 \\
2 \leq x_{11} \leq 5.5,5.5 \leq x_{12} \leq 9,4.5 \leq x_{13} \leq 12 \\
3 \leq x_{21} \leq 7,2 \leq x_{22} \leq 8,5 \leq x_{23} \leq 10 \\
1 \leq x_{31} \leq 5,4 \leq x_{32} \leq 9,2 \leq x_{33} \leq 7
\end{array}\right), \\
\bar{Z}_{R}= & 588.15, \\
\bar{Z}_{C}=493.8 & \\
\underline{Z}_{R} & =578.25 \\
\underline{Z}_{C} & =483.5 .
\end{aligned}
$$

Step 4:

$$
\begin{aligned}
& w_{R}=\frac{\overline{Z_{R}}-\underline{Z_{R}}}{\left(\overline{Z_{R}}-\underline{Z_{R}}\right)+\left(\overline{Z_{C}}-\underline{Z_{C}}\right)}=\frac{9.9}{20.2}=0.4901, \\
& w_{C}=\frac{\overline{Z_{C}}-\underline{Z_{C}}}{\left(\overline{Z_{R}}-\underline{Z_{R}}\right)+\left(\overline{Z_{C}}-\underline{Z_{C}}\right)}=\frac{10.3}{20.2}=0.50991 .
\end{aligned}
$$

Step 6: solve the following problem:

$$
\min \psi ;
$$

$$
\begin{aligned}
& {\left[\begin{array}{c}
7.9396 x_{11}+4.5579 x_{12}+10.8802 x_{13}+6.9594 x_{21}+7.4495 x_{22} \\
7.4495 x_{23}+7.4495 x_{31}+3.0876 x_{32}+5.8322 x_{33}-\psi
\end{array}\right]} \\
& {\left[\begin{array}{c}
6.6287 x_{11}+4.0027 x_{12}+9.7646 x_{13}+5.25198 x_{21}+6.5267 x_{22} \\
6.5267 x_{23}+6.5267 x_{31}+2.5495 x_{32}+5.8639 x_{33}-\psi
\end{array}\right]} \\
& x \in X^{\prime} .
\end{aligned}
$$


The solution is as follows:

$X=(3,5.5,4.5,7,2,5,4,5,6)$, and the corresponding objective value is $\left(\widetilde{Z}_{H}\right)_{\alpha}=[381.35,569.15]=475.25,93.9$, and hence

$$
\widetilde{Z}_{H}=(209,298.5,384,531.5,609.5,744) .
$$

\section{Result and Discussion}

In the Section 5, the hexagonal optimum value is $(209,298.5,384,531.5,609.5,744)$. In the optimum compromise solution, the total minimum cost will be greater than 209 and less than 744 . And as the total minimum cost lies between 384 and $531.5, \mu_{Z_{H}}$ is given as follows:

$$
\mu_{\widetilde{Z}_{H}}= \begin{cases}0, & x<209, \\ \frac{1}{2}\left(\frac{x-209}{89.5}\right), & 209 \leq x \leq 298.5, \\ \frac{1}{2}+\frac{1}{2}\left(\frac{x-298.5}{85.5}\right), & 298.5 \leq x \leq 384, \\ 1-\frac{1}{2}\left(\frac{609.5-x}{78}\right), & 531.5 \leq x \leq 609.5, \\ \frac{1}{2}\left(\frac{744-x}{134.5}\right), & 609.5 \leq x \leq 744, \\ 0, & x>744 .\end{cases}
$$

\section{Concluding Remarks}

In this paper, the capacitated transportation problem with hexagonal fuzzy costs has converted into the corresponding capacitated transportation problem with interval-valued costs and hence into the multiobjective model with two objective functions that are to minimize the right limit and the center. These two objectives are studied as the minimization of worst and average cases, respectively. The set of solution for this CTP with interval-valued costs is as the efficient solution of the corresponding multiobjective model. Therefore, the obtained efficient solution set includes the optimal compromise solutions against the worst and average cases. As a further research, we are planning to implement this solution method to solve the real-life transportation problem.

\section{Data Availability}

The data used to support the findings of this research are available from the corresponding author upon request.

\section{Conflicts of Interest}

The present address is as follows: Mathematics Department, College of Science and Arts, Al-Badaya, Qassim University, Saudi Arabia. The author declares no conflicts of interest.

\section{References}

[1] P. Pandian and G. Natarajan, "A new method for finding an optimal solution for transportation problems," International Journal of Mathematical Sciences and Engineering Applications, vol. 4, pp. 59-65, 2010.

[2] M. Oheigeartaigh, "A fuzzy transportation algorithm," Fuzzy Sets and Systems, vol. 8, pp. 235-243, 1982.

[3] S. Vajda, Readings in Linear Programming, Pitman, London, UK, 1988.

[4] P. Pandian and D. Anradha, "A new approach for solving solid transportation problems," Applied Mathematical Sciences, vol. 4, no. 72, pp. 3603-3610, 2010.

[5] P. Pandian and G. Natrajan, "An optimal more for less solution to fuzzy transportation problem with mixed constraints," Applied Mathematical Science, vol. 4, pp. 1405-1415, 2010.

[6] L. A. Zadeh, "Fuzzy sets," Information Control, vol. 8, pp. 338-353, 1965.

[7] D. Dubois and H. Prade, Fuzzy Sets and Systems; Theory and Applications, Academic Press, New York, NY, USA, 1980.

[8] M. Sakawa and H. Yano, "Interactive decision making of multi-objective nonlinear programming problems with fuzzy Parameters," Fuzzy Sets and Systems, vol. 29, pp. 315-326, 1989.

[9] S. Chanas and D. Kuchta, "A concept of the optimal solution of the transportation problem with fuzzy cost coefficients," Fuzzy Sets and Systems, vol. 82, no. 3, pp. 299-305, 1996.

[10] H. Basirzadeh, "An approach for solving fuzzy transportation problem," Applied Mathematical Sciences, vol. 7, pp. 15491566, 2011.

[11] A. Kaur and A. Kumar, "A new method for solving fuzzy transportation problems using ranking function," Applied Mathematical Modelling, vol. 35, no. 12, pp. 5652-5661, 2011.

[12] A. Kaur and A. Kumar, "A new approach for solving fuzzy transportation problems using generalized trapezoidal fuzzy numbers," Applied Soft Computing, vol. 12, no. 3, pp. 1201-1213, 2012.

[13] M. Zangiabadi and H. Maleki, "Fuzzy goal programming technique to solve multiobjective transportation problems with some non-linear membership functions," Iranian Journal of Fuzzy Systems, vol. 10, no. 1, pp. 61-74, 2013.

[14] W. S. Khalaf, "Solving fuzzy transportation problems using a new algorithm," Journal of Applied Sciences, vol. 14, no. 3, pp. 252-258, 2014.

[15] D. Dutta and P. Kumar, "Application of fuzzy goal programming approach to multi-objective linear fractional inventory model," International Journal of Systems Science, vol. 46, no. 12, pp. 2269-2278, 2015.

[16] P. Kumar and D. Dutta, "Multi-objective linear fractional inventory model of multi-products with price-dependant demand rate in fuzzy environment," International Journal of Mathematics in Operational Research, vol. 7, no. 5, pp. 547565, 2015.

[17] P. W. Shu and F. L. Deng, "Fuzzy mathematical programming approach to heterogeneous multiattribute decision-making with interval-valued intuitionistic fuzzy truth degrees," Information Sciences, vol. 325, pp. 484-503, 2015. 
[18] P. W. Shu and J. Y. Dong, "Interval-valued intuitionistic fuzzy mathematical programming method for hybrid multi-criteria group decision making with interval-valued intuitionistic fuzzy truth degrees," Information Fusion, vol. 26, pp. 49-65, 2015.

[19] D. Hunwisai and P. Kumam, "A method for solving a fuzzy transportation problem via Robust ranking technique and ATM," Cogent Mathematics, vol. 4, no. 1, 2017.

[20] R. Kumar, S. A. Edalatpanah, S. Jha, and R. Singh, "A pythagorean fuzzy approach to the transportation problem," Complex \& Intelligent Systems, vol. 5, no. 2, pp. 255-263, 2019.

[21] A. K. Bit, "Fuzzy programming with hyperbolic membership functions for multiobjective capacitated transportation problem," Opsearch, vol. 41, no. 2, pp. 106-120, 2004.

[22] M. H. Lohgaonkar and V. H. Bajaj, "Fuzzy approach to solve multi-objective capacitated transportation problem," International Journal of Bioinformatics Research, vol. 2, no. 1, pp. 10-14, 2010.

[23] K. Dahiya and V. Verma, "Capacitated transportation problem with bounds on RIM conditions," European Journal of Operational Research, vol. 178, no. 3, pp. 718-737, 2007.

[24] V. Sharma, K. Dahiya, and V. Verma, "Capacitated two-stage time minimization transportation problem," Asia-Pacific Journal of Operational Research, vol. 27, no. 4, pp. 457-476, 2010.

[25] P. W. Shu and Y. D. Jiu, "Possibility linear programming with trapezoidal fuzzy numbers," Applied Mathematical Modelling, vol. 38, pp. 1660-1672, 2014.

[26] P. W. Shu and F. L. Deng, “Atanassov's intuitionistic fuzzy programming method for heterogeneous multi-attribute group decision making with Atanassov's intuitionistic fuzzy truth degrees," IEEE Transactions on Fuzzy Systems, vol. 22, no. 2, pp. 300-312, 2014.

[27] P. W. Shu, W. Feng, G.-1. Xu, J.-Y. Dong, and T. Jing, “An intuitionistic fuzzy programming method for group decision making with interval-valued fuzzy preference relations," Fuzzy Optimization and Decision Making, vol. 16, no. 3, pp. 269-295, 2017.

[28] P. W. Shu, L. Q. Ying, and J.-Y. Dong, "A hesitant fuzzy mathematical programming method for hybrid multi-criteria group decision making with hesitant fuzzy truth degrees," Knowledge-Based Systems, vol. 138, pp. 232-248, 2017.

[29] S. Gupta, I. Ali, and A. Ahmed, "Multi-objective capacitated transportation problem with mixed constraints: a case study of certain and uncertain environment," Opsearch, vol. 55, no. 2, pp. 447-477, 2018.

[30] K. Ahmadi, "On solving capacitated transportation problem," Journal of Applied Research on Industrial Engineering, vol. 5, no. 2, pp. 131-145, 2018.

[31] P. W. Shu and J.-Y. Dong, "Pythagorean fuzzy mathematical programming method for multi-attribute group decision making with Pythagorean fuzzy truth degrees," Knowledge and Information Systems, vol. 55, pp. 437-466, 2018.

[32] J.-y. Dong and S.-P. Wan, "A new trapezoidal fuzzy linear programming method considering the acceptance degree of fuzzy constraints violated," Knowledge-Based Systems, vol. 148, pp. 100-114, 2018.

[33] J. Y. Dong and P. W. Shu, "A new method for solving fuzzy multi-objective linear programming problems," Iranian Journal of Fuzzy Systems, vol. 16, no. 3, pp. 145-159, 2019.

[34] P. Rajarajeswari, A. Sahaya Sudha, and R. Karthika, "A new operation on hexagonal fuzzy number," International Journal of Fuzzy Logic Systems, vol. 3, no. 3, pp. 15-26, 2013.
[35] A. Thamaraiselvi and R. Santhi, "Optimal solution of fuzzy transportation problem using hexagonal fuzzy numbers," International Journal of Scientific and Engineering Research, vol. 6 , no. 3, pp. 40-45, 2015.

[36] D. S. Dinagar, U. H. Narayanan, and K. Kannan, "A note on arithmetic operations of hexagonal fuzzy numbers using the $\alpha$-cut method," International Journal of Applications of Fuzzy Sets and Artificial Intelligence, vol. 1, no. 6, pp. 145-162, 2016.

[37] H. Ishibuchi and H. Tanaka, "Multiobjective programming in optimization of the interval objective function," European Journal of Operational Research, vol. 48, no. 2, pp. 219-225, 1999.

[38] M. Abd El-Hady Kassem, "Interactive stability of multi-objective nonlinear programming problems with fuzzy parameters in the constraints," Fuzzy Sets and Systems, vol. 73, no. 2, pp. 235-243, 1995. 